\title{
Weather Fluctuations May Have an Impact on Stroke Occurrence in a Society: A Population-Based Cohort Study
}

\author{
Naoki Matsumaru ${ }^{a}$ Hideshi Okadab $^{b}$ Kodai Suzuki $^{\mathrm{b}}$ Sho Nachi ${ }^{\mathrm{b}}$

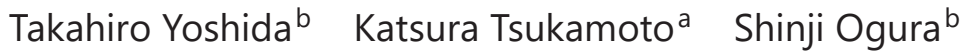 \\ aGlobal Regulatory Science, Gifu Pharmaceutical University, Gifu, Japan; \\ ${ }^{b}$ Department of Emergency and Disaster Medicine, Gifu University, Gifu, Japan
}

\section{Keywords}

Ambulance $\cdot$ Humidity $\cdot$ Stroke risk factors $\cdot$ Temperature $\cdot$ Weather

\begin{abstract}
Background: Stroke has been found to have a seasonally varying incidence; blood pressure, one of its risk factors, is influenced by humidity and temperature. The relationship between the incidence of stroke and meteorological parameters remains controversial. Objective: We investigated whether meteorological conditions are significant risk factors for stroke, focusing on the fluctuation of weather elements that triggers the onset of stroke. Methods: We collected ambulance transportation data recorded by emergency personnel from Gifu Prefecture. We included cases where the cause of the transportation was stroke and excluded cases of trauma. We combined these data with meteorological data as well as data on average temperature, average air pressure, and humidity provided publicly by the Japan Meteorological Agency. Our target period was from January 2012 to December 2016. Results: In the 5-year target period, there were 5,501 occurrences of ambulance transportation due to stroke. A seasonal tendency was confirmed, since ambulance transportation for stroke occurred more frequently at low temperatures $(p<0.001)$. Temperature (odds ratio: $0.91 ; p<0.001$ ) and humidity change (odds ratio: 1.50; $p=0.016$ ) were identified as risk factors for ambulance transportation due to stroke. An increase in temperature incurs a lower risk than a decrease (odds ratio: $0.58 ; p=0.09$ ), although there is no statistically significant difference. Conclusions: Meteorological effects on the frequency of ambulance transportation due to stroke were studied. A lower temperature and radical humidity change were identified as risk factors for ambulance transportation due to stroke, and a decrease in temperature was also associated. We speculate on the possibilities of using meteorological data to optimize the assignment of limited medical resources in medical economics.




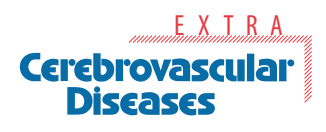

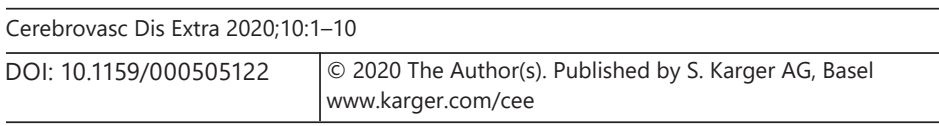

Matsumaru et al.: Impact of Weather Fluctuations on Stroke Occurrence in a Society

\section{Introduction}

Stroke is a leading cause of disability and has been classified as a high-mortality disease [1]. The risk factors for stroke are well studied, and they include high blood pressure, smoking, obesity, high cholesterol, diabetes mellitus, and atrial fibrillation [2, 3]. On the other hand, several previous reports have described a seasonally varying incidence of stroke [4-9], and ambient temperature and relative humidity were identified as risk factors for ischemic stroke onset [10]. In fact, blood pressure, a significant stroke risk factor, is known to be influenced by humidity and temperature [11], and seasonal differences in blood pressure fluctuation were observed [12]. Magalhães et al. [13] concluded that even stroke severity was associated with ambient weather conditions.

Many reports have shown that stroke incidence rates are higher during winter than during other seasons $[7,14,15]$. Some reports have found that a significantly higher seasonal incidence of hemorrhagic stroke was seen during spring and autumn $[4,5]$. Other reports have shown that the occurrence of hemorrhage is increased during the winter months $[8,16]$. Likewise, it was also reported that the frequency of ischemic stroke during summer was significantly greater than that during winter. In contrast, Mostofsky et al. [10] concluded that a lower temperature and higher levels of relative humidity increased the risk of ischemic stroke onset, regardless of the season. In other words, the relationship between the incidence of stroke and meteorological parameters remains controversial [6].

It is possible that these contrasting results are due to differences in the geographic and climatic conditions in each study and that a quantitative analysis of meteorological effects regarding stroke risk might untangle these contradictions. For example, a quantitative analysis must distinguish warming by $10^{\circ}$ from warming by $5^{\circ}$ as well as cooling by $10^{\circ}$ from cooling by $15^{\circ}$. Thus, we assumed a relationship between the fluctuation of weather elements and the onset of stroke and investigated whether the meteorological conditions were significant risk factors for stroke.

\section{Materials and Methods}

\section{Area}

The target area of this study was the Mino and South Hida area in Gifu Prefecture $\left(35^{\circ} 23^{\prime} 24.5^{\prime \prime} \mathrm{N}, 136^{\circ} 43^{\prime} 14^{\prime \prime} \mathrm{E}\right)$, located in the center of Japan with an inland climate. The climate is characterized as hot and humid in summer and cold in winter.

\section{Database}

Our data set consisted of two independent subsets. One comprised the ambulance transportation data recorded by emergency personnel of Gifu Prefecture. The target period was from January 2012 to December 2016. We included the ambulance transportation data if the reason for transportation was stroke, whereas we excluded trauma cases. The transportation data were summarized as the number of patients transported due to stroke for each day. The other data subset comprised meteorological data made publicly available by the Japan Meteorological Agency. The meteorological data were also summarized as averages for each day.

\section{Outcomes}

In this study, we defined a dichotomous outcome: the transportation frequency was either occasional or frequent in the substantive events. The categorization of transportation frequency was made based on how many patients were transported in 1 day, and the threshold was the daily average, which was 3 in our case. The transportation frequency was "occa- 
sional" if the number of transportations in 1 day was lower than the daily average. Otherwise, the transportation frequency was "frequent." We only considered the event substantive if either occasional or frequent ambulance transportation lasted for 3 consecutive days or more. In other words, instances of only 1 or 2 consecutive days were omitted, because they reflected isolated events.

\section{Explanatory Parameters}

Our explanatory parameters were the meteorological parameters. We chose the following parameters after graphical assessment of multicollinearity by scatterplot: average temperature $\left({ }^{\circ} \mathrm{C}\right)$, average barometric pressure $(\mathrm{hPa})$, and humidity $(\%)$. Additionally, we considered fluctuations or transitions in those three meteorological parameters compared to the previous day, represented by two variables: the amount and the direction of transition. A positive direction of transition indicated an increase from the previous day. The amount of transition was the absolute value of the difference. Furthermore, the time of analysis was the first day of substantive events. We focused our analysis on the first day because that was the beginning of the meaningful or nonnegligible event and we assumed that meteorological effects are substantial but slow so that manifestations of any effect may be delayed a few or even several days.

\section{Statistical Analysis}

We employed logistic regression analysis to analyze the effects of meteorological conditions on the frequency of ambulance transportation for stroke. The independent variables were the meteorological parameters on the first day of a substantive event, and the dependent variable was the binary outcome, i.e., whether transportation for stroke was occasional or frequent. We sequentially introduced groups of variables into the model, starting with temperature and the transition variates, followed by humidity and the transition variates, and finally barometric pressure and the transition variates.

Furthermore, we used a stepwise selection algorithm by AIC (Akaike information criterion) to search for an optimal model involving a statistical interaction between temperature and other meteorological parameters as interaction terms. For a reliable analysis, we restricted the number of independent variables to below $10 \%$ of the outcome events. Conformity to a linear gradient was graphically assessed, and logarithmic transformations were performed if necessary. Multicollinearity was further assessed during the statistical analysis by using the variance inflation factor (VIF), indicating serious multicollinearity if exceeding 10 and indicating a concern if greater than 4 . When involving interactions, continuous variables were substituted for the centering variables to avoid multicollinearity. All analyses were performed with R 3.6.0 at a significance level of $5 \%(p<0.05)$.

\section{Results}

There were 1,827 days in the target period of 5 years, and 5,501 stroke transportations were recorded. The average number of patients transported daily was 3.0 per day in these 5 years (3.2 for 2012, 3.1 for 2013, 2.8 for 2014, 3.0 for 2015, and 3.0 for 2016). We determined 3 as the threshold for differentiating frequent from occasional transportation. Daily ambulance transportation was frequent if there were $\geq 3$ transportations. In the target period of 5 years, $57.1 \%$ of the days $(n=1,043)$ had frequent transportation due to stroke.

Figure 1 shows the temporal changes in the frequency of daily ambulance transportations (bars) and the daily average temperatures (circles). The dotted horizontal line denotes the annual average number of transportations. The transportation frequencies seemed lower 

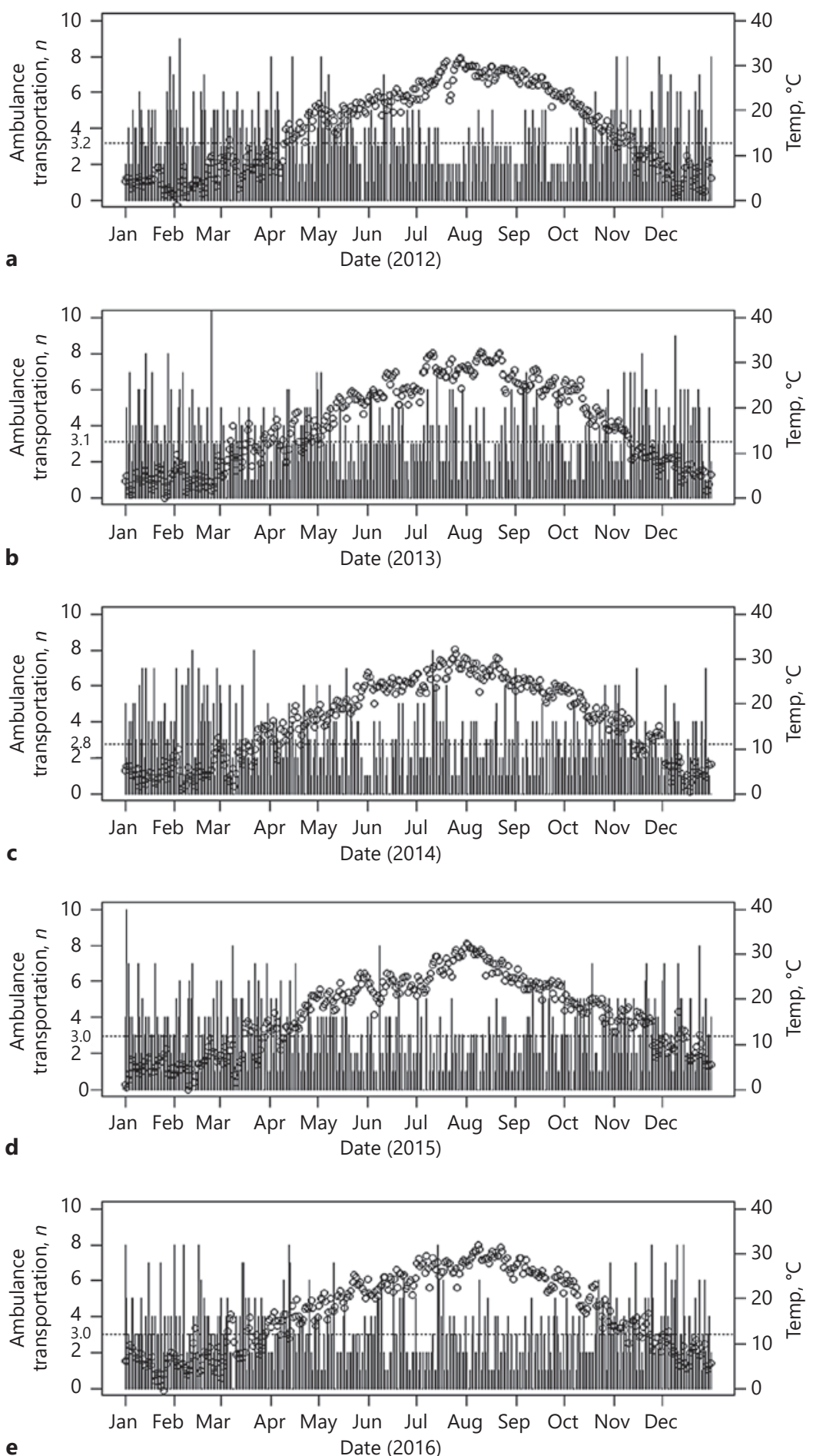

Fig. 1. Changes in the frequency of transportation by ambulance for stroke in the Gifu region (Japan) in 2012 (a), 2013 (b), 2014 (c), 2015 (d), and 2016 (e). Bars represent the number of ambulance transportations due to stroke in 1 day, and the annual average of daily frequencies is plotted with a dotted horizontal line ( 3.2 in 2012, 3.1 in 2013, 2.8 in 2014, 3.0 in 2015, and 3.0 in 2016). The average daily temperature (Temp) is indicated with circles. The transportation frequency seemingly reduced in summer with high temperatures. In winter, with low temperatures, the frequency appeared to increase. 


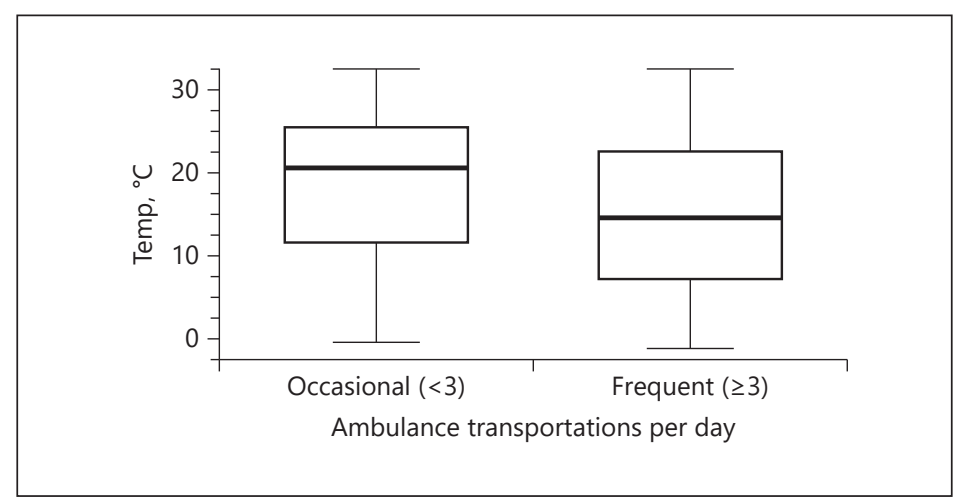

Fig. 2. Comparison of the average temperature (Temp) between days with a high frequency (frequent) and those with a low frequency (occasional) of ambulance transportation. The threshold for distinguishing frequent from occasional transportation was the average frequency of ambulance transportation $(n=3$ in this case). There was a tendency for more stroke patients to be transported in the ambulance at low temperatures, and the difference in temperature was statistically significant $\left(18.5\right.$ vs. $14.8^{\circ} \mathrm{C} ; 95 \%$ confidence interval: $2.95-4.52 ; p<0.001$ ).

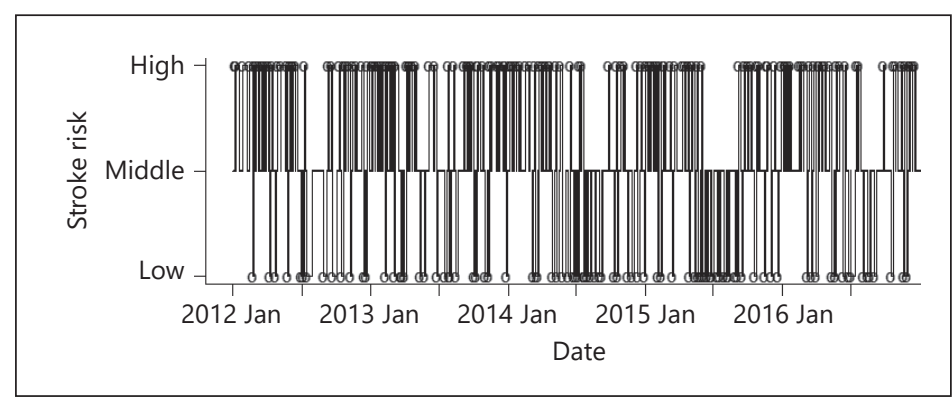

Fig. 3. Changes in stroke risk with respect to the ambulance transportation pattern. A period with a high stroke risk was defined as a period with long continuous ( $\geq 3$ days) frequent ambulance transportation. A period with a low stroke risk was defined as a period with long continuous occasional ambulance transportation. The threshold for distinguishing frequent from occasional ambulance transportation was the average frequency of ambulance transportation ( $n=3$ in this case).The risk was ambiguous or "middle" if the status of ambulance transportation frequency changed often within a short interval ( $<3$ days) between frequent and occasional.

in summer with high temperatures and vice versa in winter with low temperatures. Figure 2 compares the temperatures on days when there was occasional transportation due to stroke with those on days on which there was frequent transportation for stroke. As seen from the figure, the temperature was low when there were frequent transports, and the mean difference $\left(18.49\right.$ vs. $14.75^{\circ} \mathrm{C}$ ) was statistically significant according to the $t$ test ( $p<0.001,95 \%$ confidence interval [CI]: 2.95-4.52).

In the target period of 5 years, there were 235 substantive events covering 1,041 days (57.0\%). Out of those substantive events, 143 and 92 events were categorized as frequent and occasional, respectively. We estimated at most 9 independent variables for the logistic regression analysis for reliable analysis.

Figure 3 shows the temporal changes in meteorological stroke risk with respect to the frequency of ambulance transportation. We defined a period as "high risk" if frequent ambulance transportation continued for a substantive period of time ( $\geq 3$ days). A period with a 
Table 1. Summary of logistic regression analysis, subsequently including meteorological parameters, to elucidate effects on the frequency of ambulance transportation for stroke

\begin{tabular}{|c|c|c|c|}
\hline & \multicolumn{3}{|c|}{ Ambulance transportation for stroke, odds ratio (95\% CI) } \\
\hline & model 1 & model 2 & model 3 \\
\hline \multicolumn{4}{|l|}{ Temperature } \\
\hline Daily average $\left({ }^{\circ} \mathrm{C}\right)$ & $0.91^{* * *}(0.88,0.94)$ & $0.92^{* * *}(0.88,0.95)$ & $0.91^{* * *}(0.86,0.95)$ \\
\hline $\log _{e}(|\Delta|)$ & $1.24(0.67,2.33)$ & $1.11(0.58,2.14)$ & $1.14(0.58,2.25)$ \\
\hline Increased $(\mathrm{Y}=1 / \mathrm{N}=0)$ & $0.58 *(0.33,1.36)$ & $0.60 *(0.33,1.08)$ & $0.67(0.36,1.23)$ \\
\hline \multicolumn{4}{|l|}{ Humidity } \\
\hline Daily average (\%) & & $0.99(0.96,1.01)$ & $0.99 *(0.96,1.02)$ \\
\hline $\log _{e}(|\Delta|)$ & & $1.50^{* *}(1.08,2.11)$ & $1.50 * *(1.07,2.13)$ \\
\hline Increased $(Y=1 / N=0)$ & & $1.24(0.63,2.42)$ & $1.32(0.67,2.63)$ \\
\hline \multicolumn{4}{|l|}{ Barometric pressure } \\
\hline Daily average (hPa) & & & $0.98(0.93,1.04)$ \\
\hline $\log _{e}(|\Delta|)$ & & & $0.90(0.55,1.47)$ \\
\hline Increased $(\mathrm{Y}=1 / \mathrm{N}=0)$ & & & $1.57(0.80,3.10)$ \\
\hline AIC & 288.68 & 288.19 & 292.36 \\
\hline
\end{tabular}

Y, yes; N, no; AIC, Akaike information criterion. ${ }^{*} p<0.1,{ }^{* *} p<0.05,{ }^{* * *} p<0.01$.

"low" stroke risk indicated that ambulance transportation due to stroke was occasional for a substantive period of time. The risk was ambiguous or "middle" if the status of ambulance transportation frequency changed often within a short interval ( $<3$ days) between frequent and occasional. Our analysis excluded the middle-risk events and explored the meteorological effects in order to distinguish high from low stroke risk based on the meteorological parameters on the first day of the substantive event.

Table 1 summarizes the effects of the meteorological parameters on the frequency of ambulance transportation for stoke, based on logistic regression analysis. For the absolute amount of transition $(|\Delta|)$, natural logarithmic transformation was performed. Temperature was identified as a statistically significant factor. An increase in temperature of $1^{\circ} \mathrm{C}$ decreased the chance (odds) of frequent ambulance transportation due to stroke in one day by $9 \%$ $(\mathrm{OR}=0.91 ; 95 \% \mathrm{CI}: 0.88-0.94)$. Although it was not statistically significant, an increase in temperature reduced the odds by $42 \%(\mathrm{OR}=0.58 ; 95 \% \mathrm{CI}: 0.33-1.02 ; p=0.063)$ compared with a decrease in temperature. Including variables related to humidity in the model, another statistically significant parameter was identified, namely, the amount of humidity change. A greater "Napier's constant times humidity" change increased the odds by $50 \%$ (OR $=1.50$, 95\% CI: 1.08-2.11). Neither the humidity value nor the transition direction was identified as a statistically significant factor. When we further included three barometric pressure-related variables in the model, none of them was identified as a statistically significant factor. Evidence for multicollinearity was absent, because the VIF in all models in the table was $<3$. The maximum VIF value was 2.04 for the average barometric pressure variable in model 3.

Figure 4 shows the resulting model of stepwise selection search by AIC. The search extensively considered the interaction terms between the daily average temperature and other meteorological parameters, and the three variables were the predictors of the resulting model. The following meteorological parameters were identified as statistically significant factors regarding the frequency of the ambulance transportation due to stroke: temperature and log-transformed humidity change. The direction of temperature transition was also selected as an adjustment factor. 


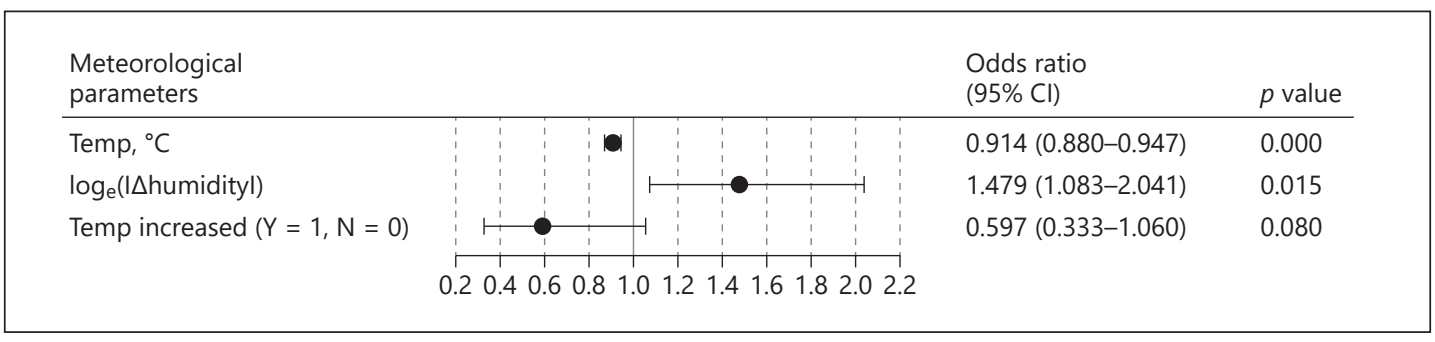

Fig. 4. Forest plot representing the odds ratio of each meteorological factor for stroke risk with respect to ambulance transportation. This model resulted from a stepwise model selection search by the AIC. The search extensively considered the interaction terms between temperature (Temp) and other meteorological parameters. Average temperature and humidity change (natural logarithmic transformation was performed) were found to be statistically significant factors for stroke risk. The temperature transition direction was also selected as a predictor, although it was not statistically significant.

\section{Discussion}

We surveyed the frequency of ambulance transportation due to stroke, and our results show that there may be a tendency towards an inverse correlation between stroke occurrence and temperature, with an increase in the number of the ambulance transportations in winter with low temperatures and a decrease in summer with high temperatures. This is consistent with the research by Anderson et al. [9], which suggested that seasonal differences in stroke risk are well known; the winter season is known to incur a higher risk for stroke than the summer season. We confirmed that the temperature value was statistically significantly different (Fig. 2) depending on whether the frequency of transportation due to stroke was occasional or frequent.

Since temperature was not the sole meteorological parameter known to have an influence on stroke $[10,11]$, we assumed that other meteorological parameters were indispensable when considering stroke risk. On the home page of the Japan Meteorological Agency, various meteorological data are available: temperature, precipitation, sunshine, snowfall, wind, humidity, vapor or barometric pressure, and cloud. In the category of temperature, for example, there are three subparameters: daily average, daily maximum, and daily minimum. Multicollinearity is apparent between those values. Some meteorological parameters are seasonal phenomena (e.g., snowfall), some are rather dichotomous (e.g., precipitation), and some are irrelevant to stroke risk (e.g., sunshine and cloud), we assumed. We targeted the following three parameters: temperature, barometric pressure, and humidity. Our choice could be arbitrary, and it might be that we should have included all the other parameters in our analysis as well. However, the number of independent variables to be included is limited. Furthermore, one of the important aspects of our analysis is the consideration of hysteresis. We hypothesized that short-term fluctuations in meteorological parameters would induce stroke or the rupture of a blood vessel. Thus, we decided to rather focus on the three abovementioned parameters instead of extensively exploring meteorological conditions.

To test our hypothesis, we defined dichotomous outcomes of the meteorological effects as frequent or occasional ambulance transportation due to stroke in a substantive event, ignoring momentary, short-term events. Some might argue that our definition of dichotomous outcomes and our focus on substantive events were simplistic and lost too much information. We believe that our simple representation of outcomes is suitable, because meteorological conditions do not necessarily have a linear effect on stroke occurrence in society. Collectively 
and socially, the consequences of meteorological conditions can be outbreaks. Effects of severe meteorological conditions can be silenced because the weather also influences people's behavior, which is a factor in stroke occurrence. Furthermore, we believe that the consequences of meteorological conditions must be neither subtle nor short-term; thus, our simple representation of data excluded individual, haphazard events.

In our analysis, we identified temperature and humidity fluctuation as significant risk factors for ambulance transportation due to stroke. Higher temperatures reduced the stroke risk, as concluded by Mostofsky et al. [10], and a radical change in humidity increased the stroke risk. Hayashi et al. [11] reported that the greater morning surge of systolic blood pressure observed in the cold seasons, as compared to the warm seasons, caused a high frequency of cardiovascular and cerebrovascular events in winter. They also observed a negative association between ambient relative humidity and systolic blood pressure. Their observation and our results imply that radical humidity fluctuation could also cause blood pressure surges and induce a high frequency of stroke events. Although it was not statistically significant, the direction of temperature transition was also selected as a predictor: an increase in temperature (e.g., in spring) incurred a lower risk than a decrease in temperature (e.g., in autumn).

We should note here as a limitation that the generalizability of our results with respect to geological and climatic differences is still uncertain. This study targeted only a specific region in a specific country, and consistent results were observed. Our key finding is that short-term weather fluctuations are also a fundamental aspect when considering biometeorological effects on stroke occurrence, although an expansion of the target region would be required for nationwide or worldwide applicability of our claim.

Analyzing meteorological effects on the frequency of ambulance transportations raises important questions regarding optimizing medical resources in medical economics. Because of the great advances in emergency medicine, mortality rates in intensive care units have lowered significantly $[17,18]$. At the same time, emergency care requires high fixed costs in terms of standby capacity; therefore, emergency departments are a focus of cost reduction efforts $[19,20]$. If it were possible to anticipate busy periods, it might be possible to prevent wasting of human resources by adjusting the medical staff or coordinating emergency hospitals within regions.

It is improbable that ambulance transportation frequencies can be predicted precisely from meteorological parameters, but we are still optimistic that the accuracy of prediction can be increased via advanced data analysis technologies (e.g., artificial intelligence and deep learning techniques). We believe that the granularity or scale of the meteorological data we assessed is appropriate for use in the management of emergency medical systems, since it is relevant to society and not only specific to individuals.

\section{Conclusions}

Combining ambulance transportation data recorded by emergency personnel with meteorological data, we elucidated meteorological effects on the frequency of ambulance transportations due to stroke. Besides low temperature, radical humidity change was identified as a factor increasing the frequency of ambulance transportation due to stroke. Employing meteorological data to predict frequencies of ambulance transportation may stimulate the discussion about how to manage regional emergency medical systems in order to optimize the use of limited resources. 

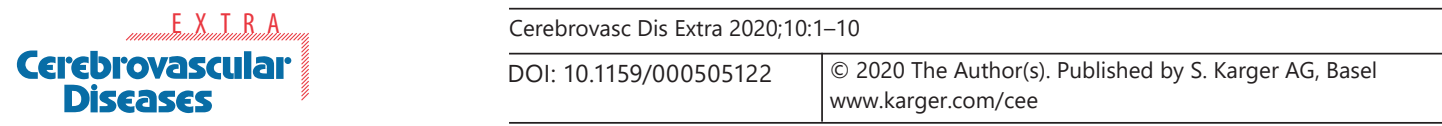

Matsumaru et al.: Impact of Weather Fluctuations on Stroke Occurrence in a Society

\section{Acknowledgements}

The authors wish to thank all the paramedical crews in Gifu Prefecture, Japan, for their service and cooperation by keeping record of their daily work. N.M. and K.T. would also like to express their gratitude to the members of the Global Regulatory Science laboratory at Gifu Pharmaceutical University for fruitful suggestions and discussions.

\section{Statement of Ethics}

Ethical approval was obtained from the medical ethics committee at the Gifu University Graduate School of Medicine (record No. 2018-104) and Gifu Pharmaceutical University, Gifu, Japan (record No. 30-29).

\section{Disclosure Statement}

The authors have no conflicts of interest to declare.

\section{Funding Sources}

No funding was received.

\section{Author Contributions}

H.O., K.T., and S.O. were substantially involved in the conception of the presented idea; N.M. and H.O. designed the study; H.O., K.S., S.N., and T.Y. were substantially involved in data acquisition; N.M. and H.O. analyzed the data and interpreted the analysis results in discussion with K.T. and S.O.; all authors read and approved the final version of the manuscript; all authors agreed to be accountable for all aspects of the work.

\section{References}

1 Towfighi A, Saver JL. Stroke declines from third to fourth leading cause of death in the United States: historical perspective and challenges ahead. Stroke. 2011 Aug;42(8):2351-5.

2 Feigin VL, Rinkel GJ, Lawes CM, Algra A, Bennett DA, van Gijn J, et al. Risk factors for subarachnoid hemorrhage: an updated systematic review of epidemiological studies. Stroke. 2005 Dec;36(12):2773-80.

3 Donnan GA, Fisher M, Macleod M, Davis SM. Stroke. Lancet. 2008 May;371(9624):1612-23.

4 Rosenørn J, Rønde F, Eskesen V, Schmidt K. Seasonal variation of aneurysmal subarachnoid haemorrhage. Acta Neurochir (Wien). 1988;93(1-2):24-7.

5 Gallerani M, Portaluppi F, Maida G, Chieregato A, Calzolari F, Trapella G, et al. Circadian and circannual rhythmicity in the occurrence of subarachnoid hemorrhage. Stroke. 1996 Oct;27(10):1793-7.

6 Hannan MA, Rahman MM, Haque A, Ahmed HU. Stroke: seasonal variation and association with hypertension. Bangladesh Med Res Counc Bull. 2001 Aug;27(2):69-78.

7 Inagawa T. Seasonal variation in the incidence of aneurysmal subarachnoid hemorrhage in hospital- and community-based studies. J Neurosurg. 2002 Mar;96(3):497-509.

8 Hakan T, Kizilkilic O, Adaletli I, Karabagli H, Kocer N, Islak C. Is there any seasonal influence in spontaneous bleeding of intracranial aneurysm and and/or AVM in Istanbul? Swiss Med Wkly. 2003 May;133(17-18):26772.

9 Anderson N, Feigin V, Bennett D, Broad J, Pledger M, Anderson C, et al. Diurnal, weekly, and seasonal variations in stroke occurrence in a population-based study in Auckland, New Zealand. N Z Med J. 2004 Sep; 117(1202):U1078. 
10 Mostofsky E, Wilker EH, Schwartz J, Zanobetti A, Gold DR, Wellenius GA, et al. Short-term changes in ambient temperature and risk of ischemic stroke. Cerebrovasc Dis Extra. 2014 Jan;4(1):9-18.

11 Hayashi T, Ohshige K, Sawai A, Yamasue K, Tochikubo O. Seasonal influence on blood pressure in elderly normotensive subjects. Hypertens Res. 2008 Mar;31(3):569-74.

12 Ohshige K, Hori Y, Tochikubo O, Sugiyama M. Influence of weather on emergency transport events coded as stroke: population-based study in Japan. Int J Biometeorol. 2006 May;50(5):305-11.

13 Magalhães R, Silva MC, Correia M, Bailey T. Are stroke occurrence and outcome related to weather parameters? Results from a population-based study in northern Portugal. Cerebrovasc Dis. 2011;32(6):542-51.

14 Walach H, Schweickhardt A, Bucher K. Does weather modify headaches? An empirical evaluation of bioweather categorization [in German]. Schmerz. 2002 Feb;16(1):1-8.

15 Fischer T, Johnsen SP, Pedersen L, Gaist D, Sørensen HT, Rothman KJ. Seasonal variation in hospitalization and case fatality of subarachnoid hemorrhage - a nationwide Danish study on 9,367 patients. Neuroepidemiology. 2005;24(1-2):32-7.

16 Nyquist PA, Brown RD Jr, Wiebers DO, Crowson CS, O’Fallon WM. Circadian and seasonal occurrence of subarachnoid and intracerebral hemorrhage. Neurology. 2001 Jan;56(2):190-3.

17 Andrews RM, Russo CA, Pancholi M. Trends in Hospital Risk-Adjusted Mortality for Select Diagnoses and Procedures, 1994-2004. Healthcare Cost and Utilization Project (HCUP) Statistical Brief \#38. Rockville (MD): Agency for Healthcare Research and Quality; 2006.

18 Hines A, Stranges E, Andrews RM. Trends in Hospital Risk-Adjusted Mortality for Select Diagnoses by Patient Subgroups, 2000-2007. Healthcare Cost and Utilization Project (HCUP) Statistical Brief \#98. Rockville (MD): Agency for Healthcare Research and Quality; 2006.

19 Pines JM, McStay F, George M, Wiler JL, McClellan M. Aligning payment reform and delivery innovation in emergency care. Am J Manag Care. 2016 Aug;22(8):515-8.

20 Gilbert SK, Wen LS, Pines JM. A comparison of perspectives on costs in emergency care among emergency department patients and residents. World J Emerg Med. 2017;8(1):39-42. 